\title{
Thermally tuneable integrated diamond micro-disk resonators fabricated by micro-assembly
}

\author{
PaUl Hill,${ }^{1 *}$ Charalambos Klitis, ${ }^{2}$ Benoit Guilhabert,${ }^{1}$ Marc Sorel,${ }^{2}$ Erdan Gu, ${ }^{1}$ \\ Martin D. Dawson, ${ }^{1}$ And Michael J. Strain. ${ }^{1}$ \\ ${ }^{I}$ Institute of Photonics, SUPA Technology and Innovation Centre, University of Strathclyde, Glasgow, UK. \\ ${ }^{2}$ School of Engineering, University of Glasgow, Glasgow, UK. \\ *corresponding address: paul.hill@strath.ac.uk
}

\begin{abstract}
We present a method for the fabrication of single crystal diamond micro-resonators and their hybrid integration with silicon waveguides. Devices with loaded Q-factors up to $\sim 7.4 \times 10^{4}$ are demonstrated in the optical fibre telecommunications C- and L-bands. Resonant wavelength tuning is achieved using thermo-optic effects at $\mathrm{mW}$ on-chip optical pump powers.
\end{abstract}

\section{Introduction}

Single crystal diamond has impressive optical properties that make it highly desirable for integrated optical applications. It is broadly transparent from the deep UV far into the infrared with a bandgap of $5.5 \mathrm{eV}$. It also exhibits strong non-linear properties with a Kerr coefficient of $13 \times 10^{-10} \mathrm{~m}^{2} / \mathrm{W}$ and a Raman gain of $\sim 10 \mathrm{~cm} / \mathrm{GW}$ at $1 \mu \mathrm{m}$. Furthermore, it plays host to several optically accessible defect centres that have shown promise as solidstate spin registers for quantum information processing $[1,2]$.

In contrast with more established optical materials, single crystal diamond is unavailable at standard waferscale, with typical chip dimensions in the mm range. Furthermore, chips often exhibit a wedged profile of $\sim 300$ $\mathrm{nm} / \mathrm{mm}[3,4]$, making it difficult to fabricate consistent optical devices across a single sample. These factors make it challenging to develop large-scale integrated photonics in diamond, and therefore a number of hybrid materials integration strategies have been developed to interface diamond with more established platforms. These include: diamond on insulator [3], thin GaP membranes integrated onto diamond [5-7]; suspended, thin, and ultrauniform diamond windows within a bulk diamond frame [8]; and, devices designed with their major axis parallel to the lowest gradient direction of a wedged diamond membrane $[9,10]$.

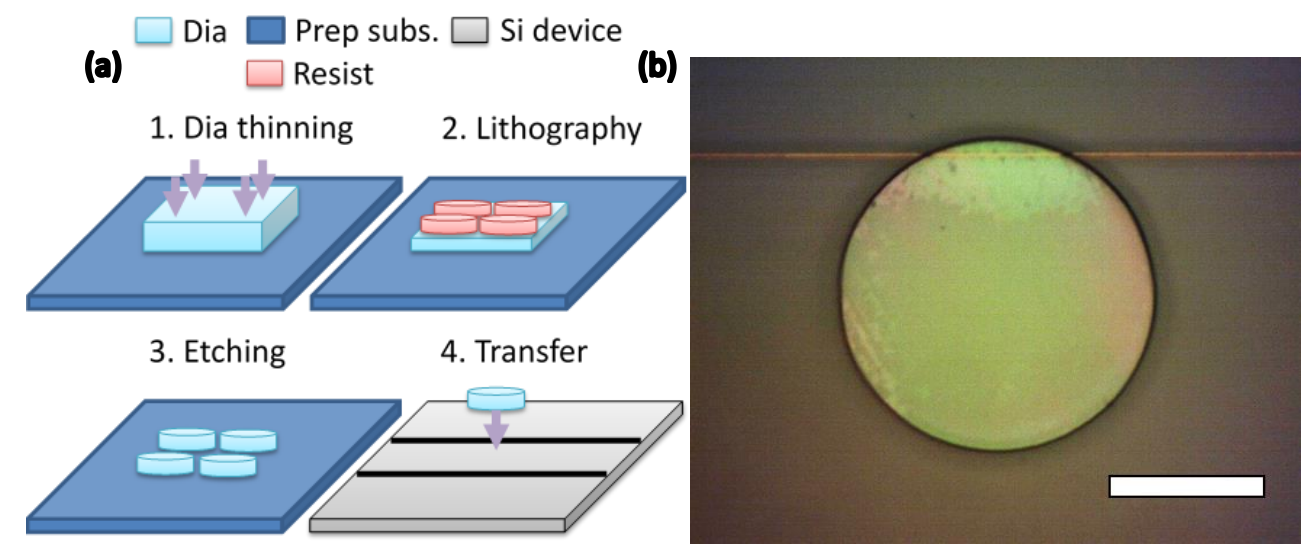

Figure 1. a) A process-flow schematic for fabrication of hybrid micro-assembled diamond resonator devices. b) A plan view optical microscope image of a fabricated device, where the scale bar is $25 \mu \mathrm{m}$.

\section{Hybrid device fabrication}

In this work the limitations of chip size are overcome in addition to offering a unique solution to the diamond chip's non-uniform thickness. Firstly, a thin diamond membrane is fabricated using inductively coupled plasma etching and then bonded to a piece of silicon for handling and further processing. Patterning of individual optical structures is achieved through e-beam lithography and subsequent plasma etching - the process flow is shown in Figure 1(a). The structures are then integrated with a host photonic integrated circuit (PIC) using transfer printing [11]. The high accuracy of the method allows precise micro-assembly of evanescently coupled resonators on a single-mode silicon waveguide platform. An example of such a hybrid diamond-silicon resonator device is shown in Figure 1(b). The transfer printing technique allows pre-selection of resonators with the correct thickness for integration, reducing the impact of the diamond chip wedge. This method also lifts the restriction on host PIC footprint defined by the diamond chip's scale, as devices can be integrated on-demand over larger areas. 


\section{Results}

The diamond micro-disk devices were integrated on a silicon waveguide chip with a spinable oxide uppercladding layer, using a transfer printing process. Transmission measurements over the optical fibre telecommunications $\mathrm{C}$ and L bands shown in Figure 2(a) and subsequent fitting of the data as in Figure 2(b) give loaded (intrinsic) Qfactors of as large as $7.4 \times 10^{4}\left(1.4 \times 10^{5}\right)$ with average loaded Q-factors around $3 \times 10^{4}\left(4.3 \times 10^{4}\right)$. The low propagation losses and high Q-factors of the resonators demonstrate their robustness to the mechanical assembly technique.
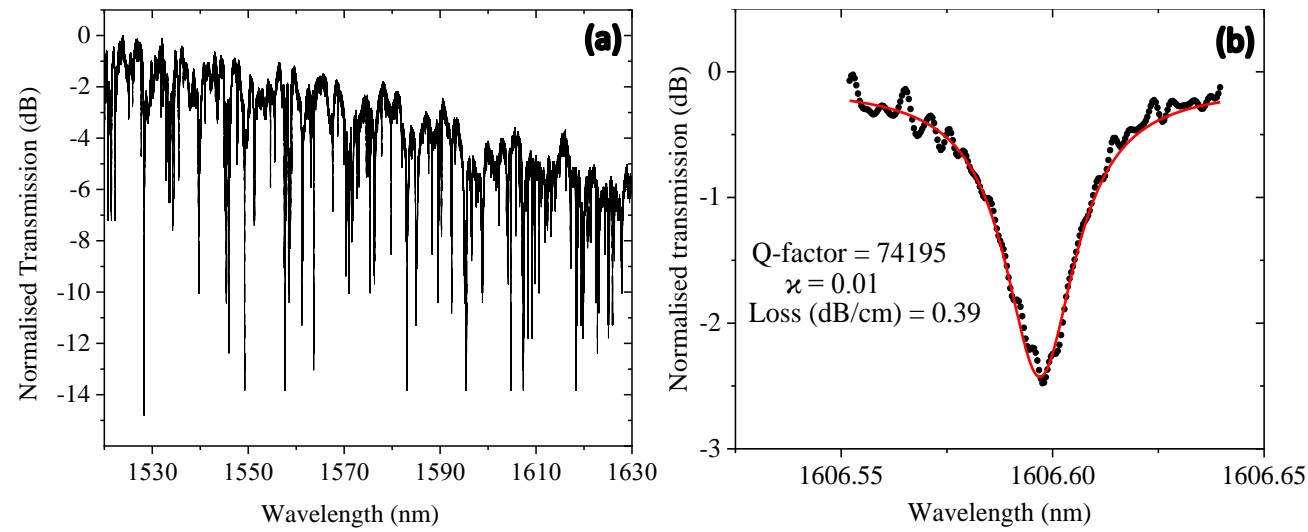

Figure 2. a) Transmission spectrum of a micro-resonator device consisting of a diamond disk coupled to a single mode silicon waveguide. b) A measured resonance of the hybrid device showing a loaded Q-factor of $\sim 74 \mathrm{k}$ along with the fit to an analytical model of a whispering gallery mode resonance .

Finally, tuning the resonant wavelengths of the device was demonstrated over a range of more than $400 \mathrm{pm}$ by optically pumping a resonance at $1563 \mathrm{~nm}$ and inducing a thermal refractive index shift of the material. Tuning was achieved with on-chip pump powers in the $\mathrm{mW}$ range and thermal bi-stability of the resonance transmission spectrum was observed as expected. The ability to optically tune resonator wavelengths by several hundred picometres on a scalable, hybrid diamond-material platform will provide a route toward the precise alignment of high Q-factor resonances and spectral features of intra-cavity emitters including color centers.

\section{Funding acknowledgements and data repository}

This work was supported by the EPSRC [EP/P013597/1], the National Quantum Information Technology Hub [EP/M013243/1], and Fraunhofer UK [EP/L015315/1].

The corresponding dataset can be found at DOI: https://doi.org/10.15129/ec085028-cd8a-4f38-8211$58 \mathrm{dfe} 5 \mathrm{~d} 62075$

\section{References}

1. I. Aharonovich, A. D. Greentree, and S. Prawer, "Diamond photonics," Nat. Photonics 5(7), 397-405 (2011).

2. P. Latawiec, V. Venkataraman, M. J. Burek, B. J. M. Hausmann, I. Bulu, and M. Lončar, "On-chip diamond Raman laser," Optica 2(11), 924 (2015).

3. B. J. M. Hausmann, I. Bulu, V. Venkataraman, P. Deotare, and M. Lončar, "Diamond nonlinear photonics," Nat. Photonics 8(5), 369-374 (2014).

4. A. H. Piracha, P. Rath, K. Ganesan, S. Kühn, W. H. P. Pernice, and S. Prawer, "Scalable Fabrication of Integrated Nanophotonic Circuits on Arrays of Thin Single Crystal Diamond Membrane Windows," Nano Lett. 16(5), 3341-3347 (2016).

5. M. Gould, E. R. Schmidgall, S. Dadgostar, F. Hatami, and K. M. C. Fu, "Efficient Extraction of Zero-Phonon-Line Photons from Single Nitrogen-Vacancy Centers in an Integrated GaP-on-Diamond Platform," Phys. Rev. Appl. 6(1), 27 (2016).

6. M. Gould, S. Chakravarthi, I. R. Christen, N. Thomas, S. Dadgostar, Y. Song, M. L. Lee, F. Hatami, and K.-M. C. Fu, "Large-scale GaP-on-diamond integrated photonics platform for NV center-based quantum information," J. Opt. Soc. Am. B 33(3), B35 (2016).

7. P. E. Barclay, K. M. C. Fu, C. Santori, A. Faraon, and R. G. Beausoleil, "Hybrid nanocavity resonant enhancement of color center emission in diamond," Phys. Rev. X 1(1), 1-7 (2011).

8. A. H. Piracha, P. Rath, K. Ganesan, S. Kühn, W. H. P. Pernice, and S. Prawer, "Scalable Fabrication of Integrated Nanophotonic Circuits on Arrays of Thin Single Crystal Diamond Membrane Windows," Nano Lett. 16(5), 3341-3347 (2016).

9. P. Latawiec, V. Venkataraman, A. Shams-Ansari, M. Markham, and M. Lončar, "Integrated diamond Raman laser pumped in the near-visible," Opt. Lett. 43(2), 318 (2018).

10. B. J. M. Hausmann, I. Bulu, V. Venkataraman, P. Deotare, and M. Lončar, "Diamond nonlinear photonics," Nat. Photonics 8(5), 369-374 (2014).

11. J. McPhillimy, B. Guilhabert, C. Klitis, M. D. Dawson, M. Sorel, and M. J. Strain, "High accuracy transfer printing of single-mode membrane silicon photonic devices," Opt. Express 26(13), 297-300 (2018). 